\title{
Finger Tapping Task Activation vs. TMS Hotspot: Different Locations and Networks
}

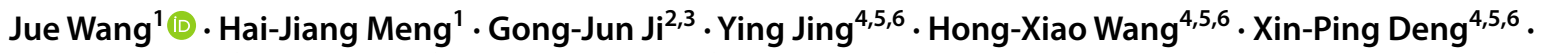 \\ Zi-Jian Feng ${ }^{4,5,6} \cdot \mathrm{Na} \mathrm{Zhao}^{4,5,6} \cdot$ Yu-Feng Zang $^{4,5,6} \cdot$ Jian Zhang ${ }^{1}$
}

Received: 18 March 2019 / Accepted: 22 October 2019 / Published online: 6 November 2019

(C) The Author(s) 2019

\begin{abstract}
Both functional magnetic resonance imaging (fMRI) and transcranial magnetic stimulation (TMS) have been used to noninvasively localize the human motor functional area. These locations can be clinically used as stimulation target of TMS treatment. However, it has been reported that the finger tapping fMRI activation and TMS hotspot were not well-overlapped. The aim of the current study was to measure the distance between the finger tapping fMRI activation and the TMS hotspot, and more importantly, to compare the network difference by using resting-state fMRI. Thirty healthy participants underwent resting-state fMRI, task fMRI, and then TMS hotspot localization. We found significant difference of locations between finger tapping fMRI activation and TMS hotspot. Specifically, the finger tapping fMRI activation was more lateral than the TMS hotspot in the premotor area. The fMRI activation peak and TMS hotspot were taken as seeds for resting-state functional connectivity analyses. Compared with TMS hotspot, finger tapping fMRI activation peak showed more intensive functional connectivity with, e.g., the bilateral premotor, insula, putamen, and right globus pallidus. The findings more intensive networks of finger tapping activation than TMS hotspot suggest that TMS treatment targeting on the fMRI activation area might result in more remote effects and would be more helpful for TMS treatment on movement disorders.
\end{abstract}

Keywords Finger tapping fMRI activation $\cdot$ TMS $\cdot$ Hotspot $\cdot$ Resting-state functional connectivity

\section{Introduction}

Handling Editor: Carlo Miniussi, Ph.D.

Yu-Feng Zang

zangyf@hznu.edu.cn

$\triangle$ Jian Zhang

zhangjian@sus.edu.cn

1 School of Psychology, Shanghai University of Sport, Shanghai 200438, China

2 Department of Medical Psychology, Chaohu Clinical Medical College, Anhui Medical University, Hefei 230032, China

3 Collaborative Innovation Centre of Neuropsychiatric Disorder and Mental Health, Hefei 230032, Anhui, China

4 Institutes of Psychological Sciences, Hangzhou Normal University, Hangzhou 311121, China

5 Zhejiang Key Laboratory for Research in Assessment of Cognitive Impairments, Hangzhou 311121, China

6 Center for Cognition and Brain Disorders and the Affiliated Hospital, Hangzhou Normal University, Hangzhou 310015, China
Transcranial magnetic stimulation (TMS) on the brain motor area has been utilized safely for decades in clinics for measuring the cortical excitability (Hanlon et al. 2015; Stinear et al. 2009), preoperative localization of motor function (Kallioniemi and Julkunen 2016; Pitkanen et al. 2015; Vitikainen et al. 2013), and repetitive TMS (rTMS) treatment for movement disorders (Wagle Shukla et al. 2016). The lateral motor cortices are among the most frequently used targets for the rTMS treatment of movement disorders, e.g., Parkinson's disease (Wagle Shukla et al. 2016), stroke (DiekhoffKrebs et al. 2017), tic disorder (Marsili et al. 2017), and writer's cramp (Havrankova et al. 2010). Vast majority of these rTMS treatment studies used the hand motor hotspot as the stimulation target. The hand motor hotspot is defined as the location where the lowest intensity evoking the highest amplitude of motor evoked potential (MEP). Although the hotspot target is a way of individualized and precise localization, there has been no strong evidence that the hotspot is an effective target for any brain disorder. That is no wonder 
why rTMS has not been approved officially (e.g., by Food and Drug Administration in the USA) for the treatment of any movement disorder.

The brain activation area of a specific task (e.g., motor task) evaluated by functional magnetic resonance imaging (fMRI) has been taken as the stimulation target for the rTMS treatment in a few studies. For example, a study utilized a working memory task and defined fMRI activation in the dorsal lateral prefrontal cortex (DLPFC) as the stimulation target of rTMS treatment on multiple sclerosis (Hulst et al. 2017). However, few studies have used motor task fMRI activation as rTMS target in the lateral motor cortices. One reason might be that rTMS studies targeting on the motor cortices usually focused on movement disorder, and many patients with movement disorder have disability to perform motor task. Another possible reason is that the vast majority rTMS studies targeting on the hotspot claimed to stimulate the primary motor cortex (M1). These studies might have considered the hotspot and fMRI motor activation area the similar areas.

The primary motor area was structurally defined with the hand representation areas buried within the central sulcus and rarely extending to the gyral surface (Geyer et al. 1996). But the case is more complicated for fMRI motor activation studies and TMS hotspot studies. Many TMS studies considered the hotspot as the primary motor area (Benninger et al. 2011; Benninger et al. 2009; Khedr et al. 2003, 2006; Siebner et al. 2000) or hand knob (hand movement activation area, "knob-shaped" curvature of the precentral gyrus (Puce et al. 1995; Yousry et al. 1997)). However, an MRIguided TMS study found that the hotspot was located at the pre-motor area, i.e., anterior to the hand knob (Ahdab et al. 2016). A few studies combined TMS and motor task fMRI and found that the hotspot was not overlapped with the motor task activation area (Bastings et al. 1998; Diekhoff et al. 2011; Herwig et al. 2002; Lotze et al. 2003b; Sparing et al. 2008).

The hotspot location is determined by MEP which reflects the conduction of the descending corticospinal or corticonuclear pyramidal tract (Lefaucheur 2019), while the fMRI activation reflects the hemodynamic changes when response to motor tasks (Bastings et al. 1998). The TMS and fMRI techniques provide related but different information. These two locations may have different underlying neural networks. Resting-state fMRI (RSfMRI) seed-based functional connectivity is a systemslevel approach to assess the relationship between the seed region and other brain areas. It represents the temporal synchronization of spontaneous brain activity among distributed brain regions associated with particular functions (Cole et al. 2010; Fox et al. 2005; Hampson et al. 2002; Van Dijk et al. 2010). A study successfully separated motor from sensory components by using functional connectivity, although the distance of these two cortices was very close (Zhang et al. 2009). RS-fMRI studies have shown that stimulating on the superficial target could affect the functional connectivity on remote brain areas (Eldaief et al. 2011; Wang et al. 2014) and hence improved memory performance (Wang et al. 2014).

The first aim of the current study was to explore the difference of the location of TMS hotspot and task fMRI activation in the precentral gyrus. More importantly, the second aim was to investigate the functional connectivity network differences of these locations. Since the finger tapping task required more active processing than TMSinduced finger movement, we hypothesized that the finger tapping activation area would have more intensive functional connectivity. If so, the results of the current study would help choose the stimulation target on the motor cortex for rTMS treatment of movement disorders.

\section{Materials and Methods}

\section{Participants}

Thirty right-handed healthy participants (16 females, 18-28 years old, mean age \pm standard deviation: $22.9 \mathrm{y} \pm 2.7$ ) were recruited by internet advertisement. All participants had no history of head trauma, substance abuse, or neuropsychiatric disorders. The whole study was carried out and approved by the Ethics Committee of the Center for Cognition and Brain Disorders (CCBD) at Hangzhou Normal University (HZNU). Informed consent was obtained from all individual participants included in the study.

\section{Experimental Design}

Participants underwent a resting-state fMRI scan session and then a task fMRI session. Then they went to the TMS room.

In the resting-state fMRI session, participates were asked to keep their eyes closed, relax, remain as motionless as possible, not think of anything in particular, and not fall asleep. Foam pads were used to ensure the head comfortably and minimize the head motion.

During the task fMRI session, participants were asked to perform a block design task of right thumb tapping during a 4-min scanning in MRI scanner. Participants placed their right hands palm uppermost and held a button in palm, then pressed the button with their right thumb when a picture of thumb appears in the center of the screen. The picture lasted $500 \mathrm{~ms}$ then followed a 1500-ms fixation. Each block lasted $40 \mathrm{~s}$ and there were 6 blocks in total (Fig. 1). 


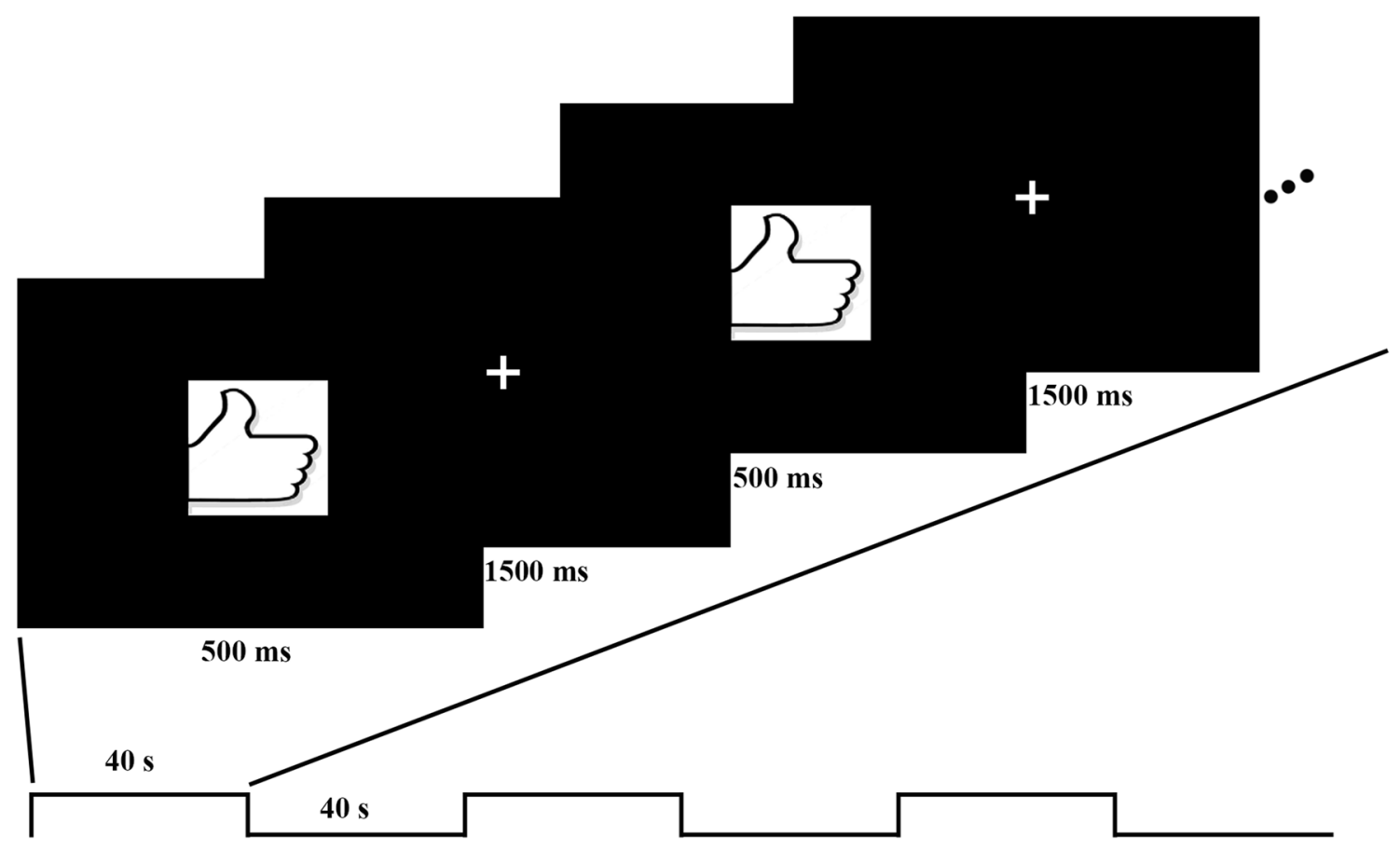

Fig. 1 The block design task with right thumb tapping, during a 4-min scanning in MRI scanner

\section{Data Acquisition}

\section{MRI Data Acquisition}

MRI data were acquired on a GE 3T scanner (MR-750, GE Medical Systems, Milwaukee, WI) at the Center for Cognition and Brain Disorders (CCBD) of Hangzhou normal university. The fMRI scanning sessions using gradient-echo echo planar imaging (EPI) sequences included an 8-min resting-state session and a 4-mintask session with the following parameters: repetition time $(\mathrm{TR})=2000 \mathrm{~ms}$, echo time $(\mathrm{TE})=30 \mathrm{~ms}$, flip angle $(\mathrm{FA})=90^{\circ}, 43$ slices with no gap, matrix $=64 \times 64$, field of view $(\mathrm{FOV})=220 \times 220 \mathrm{~mm}$, acquisition voxel size $=3.44 \times 3.44 \times 3.2 \mathrm{~mm}$. A high resolution $\mathrm{T} 1$ anatomical image was scanned for accurate localization (176 sagittal slices, thickness $=1 \mathrm{~mm}, \mathrm{TR}=8.1 \mathrm{~ms}$, $\left.\mathrm{TE}=3.1 \mathrm{~ms}, \mathrm{FA}=8^{\circ}, \mathrm{FOV}=250 \times 250 \mathrm{~mm}\right)$.

\section{Electromyographic Recording}

Surface electromyogram (EMG) was used to record the MEP amplitudes of the right abductor pollicis brevis (APB) muscle using 9-mm diameter $\mathrm{Ag} / \mathrm{AgCl}$ surface electrodes with their centers 20-30 mm apart over the muscle bellies. The active electrode was placed over the venter musculi and the reference electrode was placed over metacarpophalangeal joint. The EMG signal of APB muscle was amplified $(1000 \times)$, bandpass filtered $(20 \mathrm{~Hz}-2.5 \mathrm{kHz}$; Intronix Technologies Model 2024F) and digitized at $5 \mathrm{kHz}$ by an analog-to-digital interface (Micro 1401; Cambridge Electronics Design, Cambridge, UK), then saved in a computer and projected on a screen.

\section{TMS Hotspot Measurement}

Single pulse TMS (Magstim Co., Wales, UK) was employed with a figure- 8 coil (diameter $=70 \mathrm{~mm}$ ). Participants sat in a cozy chair with both arms relaxed on their thighs. Through visual and EMG monitoring, full muscle relaxation was ensured. The coil was firstly placed over the left M1 (hand knob) with the handle backwards and $45^{\circ}$ against the sagittal midline of the brain for measuring the MEP in the target muscle. The coil orientation was monitored by BrainSight TMS navigation system (Rogue Research, Montreal, Canada) to keep it always perpendicular to the central sulcus and tangential to the scalp (Rossini et al. 2015). The hand motor hotspot was defined as the location around the "hand knob" where the lowest intensity evoking the highest amplitude of MEPs. To determine the hotspot, the coil was then shifted every $0.5 \mathrm{~cm}$ each time around the hand knob. The resting motor threshold (RMT) was quantified as the lowest intensity evoking a response $(>50 \mu \mathrm{V})$ in more than 5 of 10 consecutive trials. The stimulus intensity for all participants was lower than $50 \%$ of the maximal stimulator output (range $27-48 \%$, mean \pm stander deviation $38 \% \pm 0.05$ ). The number of stimuli for each position was maximal to 10 . If the hotspot could not be determined within 10 stimuli, then the coil was moved to the next position. Finally, the hotspot of 
APB muscle was marked with BrainSight TMS navigation system on individual structure MRI images.

\section{Data Analyses}

The fMRI data preprocessing of both task and resting-state was conducted by using DPABI_V3.0 (http://rfmri.org/dpabi ) software (Yan et al. 2016).

\section{Task fMRI Data Preprocessing}

The task data preprocessing included the following steps: (1) correcting for the acquisition time delay between slices, (2) rigid-body realigning for estimation and correction of the motion displacement (all participants' head motion less than $1 \mathrm{~mm}$ in translation or 1 degree in rotation in any direction), (3) co-registering the functional images to T1 image and then normalizing to MNI space using the echo-planar imaging (EPI) template in statistical parametric mapping 12 (SPM12, https://www.fil.ion.ucl.ac.uk/spm/software/spm12 ), (4) spatial smoothing with a Gaussian kernel of 6-mm full width at half maxima (FWHM).

\section{Resting-State fMRI Preprocessing}

The resting-state fMRI data preprocessing included: (1) discarding the first 10 volumes to allow the signal to reach equilibrium and the subjects to adapt to the scanning noise, (2) correcting for the acquisition time delay between slices, (3) rigid-body realigning for estimation and correction of the motion displacement (all participants' head motion less than $1 \mathrm{~mm}$ in translation or 1 degree in rotation in any direction), (4) normalizing to MNI space using the EPI template in SPM12, (5) regressing out nuisance signals (white matter, cerebrospinal fluid signals, and 24 head-motion parameters (Yan et al. 2013)), (6) removing the linear trend, (7) band-pass (0.01-0.08 Hz) filtering, and (8) smoothing with a Gaussian kernel of 6-mm FWHM.

\section{Statistical Analysis}

\section{Task fMRI Activation Detection}

SPM12 was used for subject-level activation analysis (high-pass filtering, $>1 / 128 \mathrm{~Hz}$, was selected in "fMRI Model specification") and group-level statistical analysis. The group-level activation map (Fig. 2, FDR correction, $\mathrm{Q}<0.01$ ) was saved as Mask-1. The left precentral gyrus of Harvard-Oxford atlas in FSL (http://www.fmrib.ox.ac.uk/ fsl) was extracted as Mask-2. Then an intersection mask of Mask-1 and Mask-2 (Fig. 3) was generated to restrict the location of activation peak voxel of each participant.

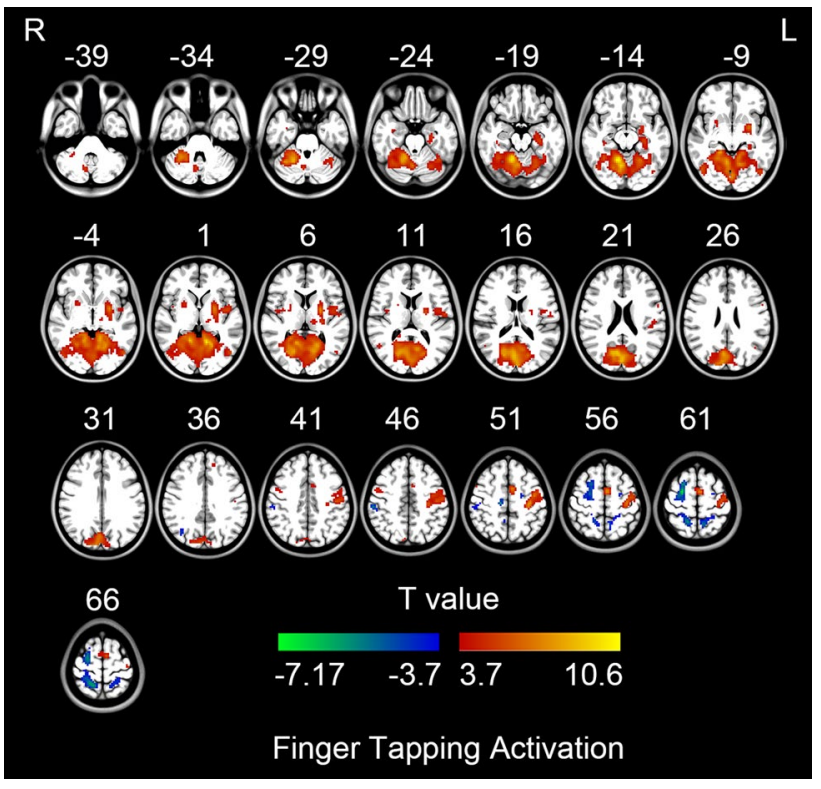

Fig. 2 Group level finger tapping activation map (FDR correction, $\mathrm{Q}<0.01)$. The warm color indicates the activation of finger tapping task; the cold color indicates the deactivation of finger tapping task. $\mathrm{L}$, left hemisphere; R, right hemisphere

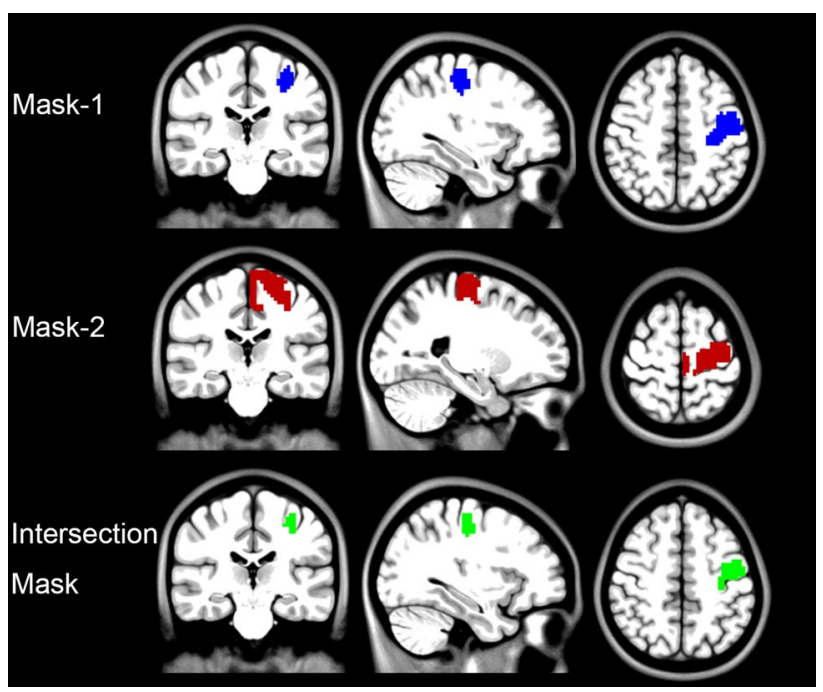

Fig. 3 Generation of an intersection mask for the definition of the task activation peak voxel of each participant. The result of group level activation was saved as Mask-1, and the left precentral gyrus of Harvard-Oxford atlas was extracted as Mask-2. Then the intersection mask was generated by Mask-1 and Mask-2

\section{Locations of Task fMRI vs. TMS Hotspot in the Precentral Gyrus}

The peak voxel of each participant in the intersection mask was considered as the presentation of finger tapping task (Table 1). The MNI coordinates of hotspot for APB were 
Table 1 The information of peak voxels of finger tapping activation in the intersection mask

\begin{tabular}{|c|c|c|c|c|c|c|}
\hline \multirow{2}{*}{$\begin{array}{l}\text { Subject ID } \\
\text { Sub001 }\end{array}$} & \multirow{2}{*}{$\begin{array}{l}\text { Brodmann area } \\
6\end{array}$} & \multicolumn{3}{|c|}{ Coordinate (X Y Z) } & \multirow{2}{*}{$\begin{array}{l}\mathrm{T} \text { value } \\
3.65\end{array}$} & \multirow{2}{*}{$\begin{array}{l}\begin{array}{l}p \text { Value } \\
\text { (uncor- } \\
\text { rected) }\end{array} \\
<0.001\end{array}$} \\
\hline & & -54 & 3 & 42 & & \\
\hline Sub002 & 6 & -48 & -3 & 51 & 5.87 & $<0.001$ \\
\hline Sub003 & 6 & -51 & -12 & 54 & 7.13 & $<0.001$ \\
\hline Sub004 & 4 & -51 & -12 & 45 & 5.28 & $<0.001$ \\
\hline Sub005 & 6 & -45 & 6 & 42 & 5.22 & $<0.001$ \\
\hline Sub006 & 4 & -39 & -18 & 54 & 3.80 & $<0.001$ \\
\hline Sub007 & 4 & -36 & -18 & 51 & 5.75 & $<0.001$ \\
\hline Sub008 & 4 & -42 & -18 & 51 & 4.33 & $<0.001$ \\
\hline Sub009 & 6 & -39 & -15 & 54 & 4.68 & $<0.001$ \\
\hline Sub010 & 4 & -51 & -9 & 45 & 6.49 & $<0.001$ \\
\hline Sub011 & 6 & -48 & -3 & 48 & 1.50 & $<1$ \\
\hline Sub012 & 6 & -48 & -3 & 51 & 4.90 & $<0.001$ \\
\hline Sub013 & 4 & -36 & -18 & 54 & 4.12 & $<0.001$ \\
\hline Sub014 & 6 & -42 & -6 & 57 & 6.53 & $<0.001$ \\
\hline Sub015 & 6 & -42 & -6 & 57 & 7.81 & $<0.001$ \\
\hline Sub016 & 4 & -42 & -18 & 54 & 8.07 & $<0.001$ \\
\hline Sub017 & 6 & -48 & 0 & 51 & 1.59 & $<0.1$ \\
\hline Sub018 & 6 & -36 & -12 & 54 & 6.09 & $<0.001$ \\
\hline Sub019 & 6 & -51 & -9 & 48 & 4.77 & $<0.001$ \\
\hline Sub020 & 6 & -54 & -3 & 45 & 3.65 & $<0.001$ \\
\hline Sub021 & 3 & -39 & -21 & 51 & 3.77 & $<0.001$ \\
\hline Sub022 & 4 & -39 & -21 & 54 & 8.28 & $<0.001$ \\
\hline Sub023 & 6 & -42 & -6 & 57 & 3.05 & $<0.005$ \\
\hline Sub024 & 6 & -42 & -3 & 48 & 3.90 & $<0.001$ \\
\hline Sub025 & 6 & -54 & 0 & 42 & 2.01 & $<0.05$ \\
\hline Sub026 & 4 & -51 & -12 & 45 & 5.59 & $<0.001$ \\
\hline Sub027 & 6 & -42 & -3 & 57 & 4.02 & $<0.001$ \\
\hline Sub028 & 6 & -36 & -15 & 60 & 7.22 & $<0.001$ \\
\hline Sub029 & 6 & -42 & -6 & 57 & 3.43 & $<0.001$ \\
\hline Sub030 & 4 & -48 & -12 & 42 & 4.49 & $<0.001$ \\
\hline Mean & & -45 & -9 & 51 & & \\
\hline
\end{tabular}

exported from BrainSight system. It should be noted that these coordinates were all located above the scalp. We used a home-made toolkit TMStarget (http://www.brainhealthy.net) (Ji et al. 2017) to convert the exported scalp coordinates to cortex coordinates as follows. First, the structural images were segmented and spatially normalized by "new segment" in SPM12, and the normalized grey matter images were thresholded with a grey matter probability $>0.2$. Second, the Euclidean distance between the scalp coordinates and the surface of cortices were estimated. Accordingly, the spot of Euclidean distance from the scalp coordinate was marked, and then went deep $6 \mathrm{~mm}$ further along the line which is perpendicular to the tangent passing through the spot. Finally, the location for each subject (Table 2) were determined as the presentation of TMS-induced movement of APB in the brain (Please see the Diagram of Fig. 4). These coordinates were employed to compare the difference of locations between fMRI finger tapping activation and TMS-induced movement by using SPSS (https://www.ibm.com/analytics/ spss-statistics-software).

\section{Resting-State fMRI Functional Connectivity}

The coordinates of fMRI finger movement peak voxel in Table 1 and TMS-induced movement presentations in Table 2 were taken as seeds for computing voxel-wise functional connectivity of resting-state fMRI data. For each participant, the mean time course of a sphere (radius $4 \mathrm{~mm}$ ) centered at the seed coordinate was acquired, then the functional connectivity was calculated. One-sample t-tests were performed at group level for each type of movement. Voxels above a corrected threshold (FDR correction, $\mathrm{Q}<0.0001$ and $\mathrm{Q}<0.000000005$ ) 
Table 2 The coordinates of TMS APB hotspot

\begin{tabular}{|c|c|c|c|c|}
\hline \multirow{2}{*}{$\begin{array}{l}\text { Subject ID } \\
\text { Sub001 }\end{array}$} & \multirow{2}{*}{$\begin{array}{l}\text { Brodmann area } \\
6\end{array}$} & \multicolumn{3}{|c|}{$\begin{array}{l}\text { Montreal Neurological } \\
\text { Institute (X Y Z) }\end{array}$} \\
\hline & & -36 & -18 & 66 \\
\hline Sub002 & 6 & -24 & -18 & 69 \\
\hline Sub003 & 6 & -28 & -2 & 67 \\
\hline Sub004 & 4 & -47 & -18 & 59 \\
\hline Sub005 & 6 & -37 & -12 & 65 \\
\hline Sub006 & 6 & -40 & -6 & 63 \\
\hline Sub007 & 4 & -45 & -11 & 64 \\
\hline Sub008 & 6 & -43 & 0 & 56 \\
\hline Sub009 & 6 & -38 & -8 & 62 \\
\hline Sub010 & 4 & -38 & -19 & 65 \\
\hline Sub011 & 6 & -40 & -8 & 63 \\
\hline Sub012 & 6 & -33 & -7 & 66 \\
\hline Sub013 & 3 & -52 & -22 & 54 \\
\hline Sub014 & 6 & -50 & -4 & 55 \\
\hline Sub015 & 6 & -25 & -3 & 68 \\
\hline Sub016 & 6 & -18 & -6 & 69 \\
\hline Sub017 & 4 & -45 & -16 & 67 \\
\hline Sub018 & 6 & -41 & -11 & 65 \\
\hline Sub019 & 6 & -34 & -14 & 68 \\
\hline Sub020 & 6 & -35 & -15 & 64 \\
\hline Sub021 & 6 & -42 & -9 & 63 \\
\hline Sub022 & 4 & -42 & -14 & 66 \\
\hline Sub023 & 6 & -38 & -19 & 67 \\
\hline Sub024 & 6 & -31 & -12 & 68 \\
\hline Sub025 & 6 & -42 & -12 & 64 \\
\hline Sub026 & 6 & -28 & -14 & 70 \\
\hline Sub027 & 6 & -38 & -16 & 65 \\
\hline Sub028 & 6 & -19 & -18 & 71 \\
\hline Sub029 & 3 & -43 & -29 & 56 \\
\hline Sub030 & 6 & -46 & -12 & 60 \\
\hline Mean & & -37 & -12 & 64 \\
\hline
\end{tabular}

The coordinate of $\mathrm{Z}$ axis was estimated. Please see the Materials and methods section, "Locations of task fMRI vs. TMS hotspot in the precentral gyrus" for details

$T M S$ transcranial magnetic stimulation, $A P B$ abductor pollicis brevis

were taken as showing significant functional connectivity with the seed region. Paired t-tests were conducted between the two types of functional connectivity maps throughout the whole brain. Voxels above corrected threshold (GRF correction, single voxel $\mathrm{p}<0.001$, cluster level $\mathrm{p}<0.05)$ were taken as showing significant different functional connectivity between the two types of movement.

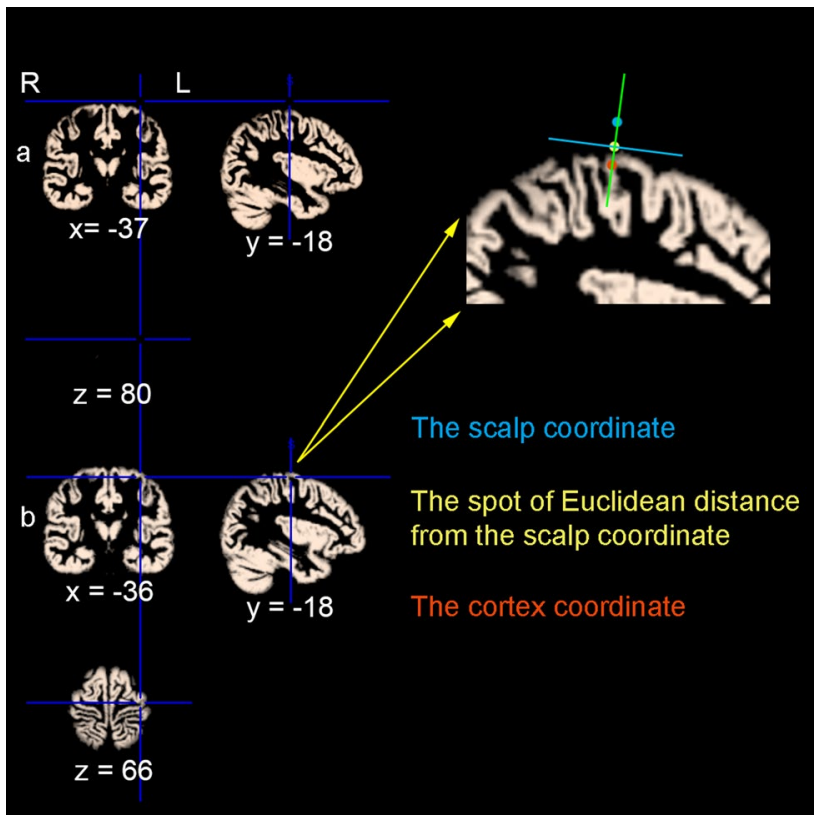

Fig. 4 Diagram of the converting procedure from scalp coordinate to cortex coordinate (an example from a participant). The segmented spatially normalized structural image was thresholded with a grey matter probability $>0.2$. a The MNI coordinate of hotspot for APB was exported from BrainSight system. b The converted cortex coordinate. The blue dot indicates the scalp coordinate, the yellow dot indicates the spot of Euclidean distance from the scalp coordinate, and the red dot indicates the cortex coordinate. The distance between yellow dot and red dot is $6 \mathrm{~mm}$. The green line indicates the actual direction of coil which is perpendicular to the tangent (the blue line) passing through the spot

\section{Results}

\section{The Distance Between fMRI Finger Tapping Activations and TMS Hotspot}

Paired t-tests showed that there were significant differences in $\mathrm{X}$ and $\mathrm{Z}$ axes (Bonferroni correction, 0.05/3 $=0.0167$, the actual $\mathrm{p}<0.001$, Table 3 ). The thumb tapping activations sat

Table 3 The differences of spatial localizations (finger tapping activation vs. APB hotspot)

\begin{tabular}{llll}
\hline & \multicolumn{3}{l}{ Mean coordinate $(\mathrm{mm}) \pm$ standard deviation } \\
\cline { 2 - 4 } & $\mathrm{x}$ & $\mathrm{y}$ & $\mathrm{z}$ \\
\hline Activation peak & $-44.6 \pm 5.9$ & $-9.1 \pm 7.4$ & $50.7 \pm 5.3$ \\
APB hotspot & $-37.3 \pm 8.5$ & $-12.4 \pm 6.5$ & $64.2 \pm 4.5$ \\
T value & -3.7 & 1.7 & -10.5 \\
$\mathrm{p}$ Value & $0.001 *$ & 0.102 & $<0.001 *$ \\
\hline
\end{tabular}

$A P B$ abductor pollicis brevis

*Bonferroni correction 
more laterally and more inferior ( $\mathrm{X}$ and $\mathrm{Z}$ axes, respectively. Table 3). Both thumb tapping activation and TMS-induced movement representations were not at "hand knob" (Fig. 5).

\section{Brain Areas of Finger Tapping Task fMRI Activation}

The right thumb tapping task activated extensive brain areas including the bilateral putamen, bilateral insula, ipsilateral precentral gyrus, cerebellum, visual area, contralateral

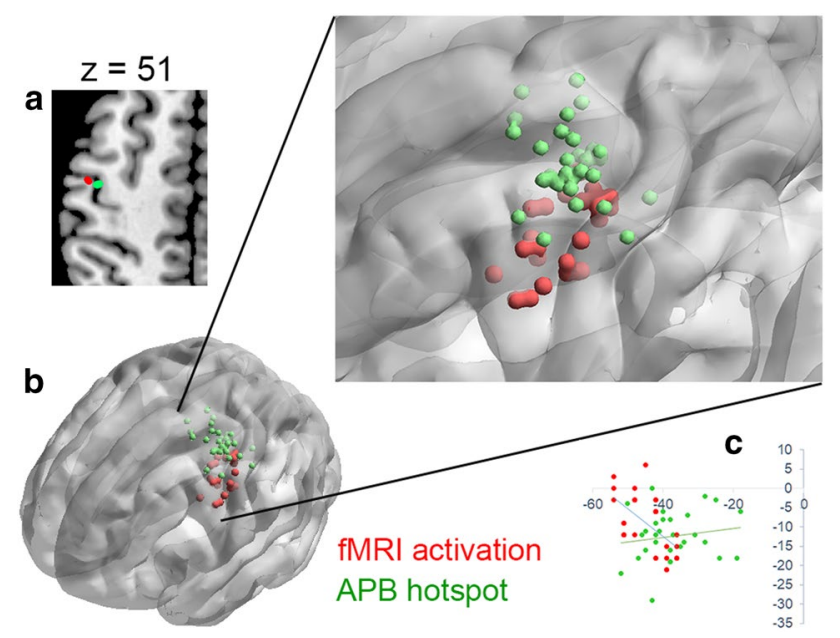

Fig. 5 Localization of the premotor area with fMRI activation and TMS hotspot. a The red ellipsoid indicates mean MNI coordinates $( \pm$ SD) of fMRI activation peak voxel, and the green ellipsoid indicates mean MNI coordinates $( \pm \mathrm{SD})$ of the TMS hotspot. All these coordinates sat in left hemisphere. $\mathbf{b}$ Individual locations project to a three-dimensional brain template. c A scatter diagram of individual locations precentral gyrus, supplementary motor area, and thalamus (Fig. 2, Table 4).

\section{Functional Connectivity Results}

The activation-based and APB hotspot-based functional connectivity maps were shown in Fig. 6 (Fig. 6 upper row). Each of the two types of seeds showed extensive functional connectivity with the bilateral sensorimotor area, occipital area, cerebellum, and prefrontal gyrus. Their maps looked similar by visual inspection. Under a rigorous threshold, the different seeds showed very different functional connectivity patterns throughout the whole brain by visual inspection (Fig. 6 lower row).

Paired t-tests showed that the finger tapping activation seeds had significantly more intensive functional connectivity than TMS-induced seeds, including the right globus pallidus, bilateral putamen, bilateral insula, and bilateral precentral gyri (Fig. 7, Table 5). No any significantly more intensive functional connectivity was found for TMSinduced than that of finger tapping activation seed.

\section{Discussion}

\section{Different Locations of Finger Tapping fMRI Activation and TMS Hotspot}

The current study found a spatial mismatch, i.e., more lateral fMRI activation than the hotspot, of the motor cortical locations between finger tapping fMRI activation and TMS-induced finger movement (hotspot), being consistent

Table 4 Finger tapping activations in the whole brain

\begin{tabular}{|c|c|c|c|c|c|c|c|}
\hline Brain region & Brodmann area & Montreal Neurological & Institut & X Y Z) & Cluster size $\left(\mathrm{mm}^{3}\right)$ & $\mathrm{T}$ value & $Q$ value \\
\hline Right Occipital Lobe & 19 & 12 & -54 & -15 & 126,198 & 10.60 & $<0.01$ \\
\hline Right Hippocampus & 20 & 33 & -3 & -27 & 216 & 4.62 & $<0.01$ \\
\hline Left Putamen & & -24 & -3 & 6 & 13,554 & 7.40 & $<0.01$ \\
\hline Right Hippocampus & 20 & 36 & -27 & -15 & 378 & 4.18 & $<0.01$ \\
\hline Right Putamen & & 24 & 6 & 0 & 1350 & 4.67 & $<0.01$ \\
\hline Left Thalamus & & -12 & -21 & 6 & 621 & 4.63 & $<0.01$ \\
\hline Right Insula & 48 & 36 & -3 & 9 & 810 & 4.22 & $<0.01$ \\
\hline Right Lateral Occipital Cortex & 37 & 51 & -60 & 12 & 189 & 4.45 & $<0.01$ \\
\hline Left Postcentral Gyrus & 48 & -51 & -21 & 21 & 486 & 4.27 & $<0.01$ \\
\hline Left Precentral Gyrus & 6 & -57 & 6 & 24 & 135 & 4.64 & $<0.01$ \\
\hline Left Angular Gyrus & 39 & -48 & -66 & 24 & 162 & 3.98 & $<0.01$ \\
\hline Left Precentral Gyrus & 4 & -33 & -21 & 48 & 9396 & 7.49 & $<0.01$ \\
\hline Left Frontal Pole & 32 & -12 & 42 & 39 & 351 & 4.30 & $<0.01$ \\
\hline Left Supplementary Motor Area & 6 & -6 & 0 & 54 & 3834 & 8.39 & $<0.01$ \\
\hline Right Precentral Gyrus & 6 & 51 & 0 & 45 & 648 & 4.67 & $<0.01$ \\
\hline
\end{tabular}

$Q$ value false discovery rate (FDR) correction 
Fig. 6 The activation-based and APB hotspot-based whole brain functional connectivity maps (FDR correction, $\mathrm{Q}<0.0001$ for upper row, $\mathrm{Q}<0.000000005$ for lower row). L, left hemisphere; $\mathrm{R}$, right hemisphere; FC, functional connectivity

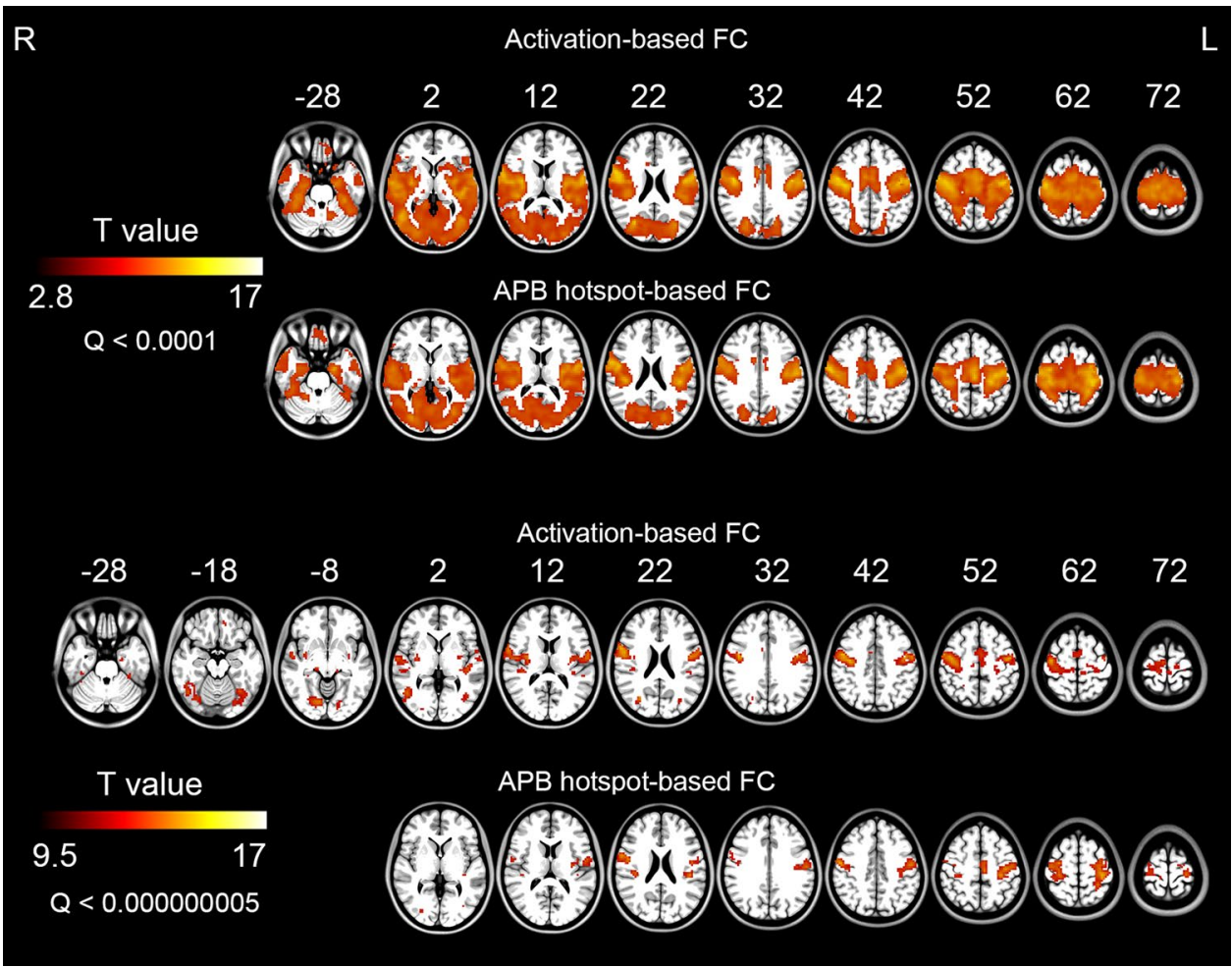

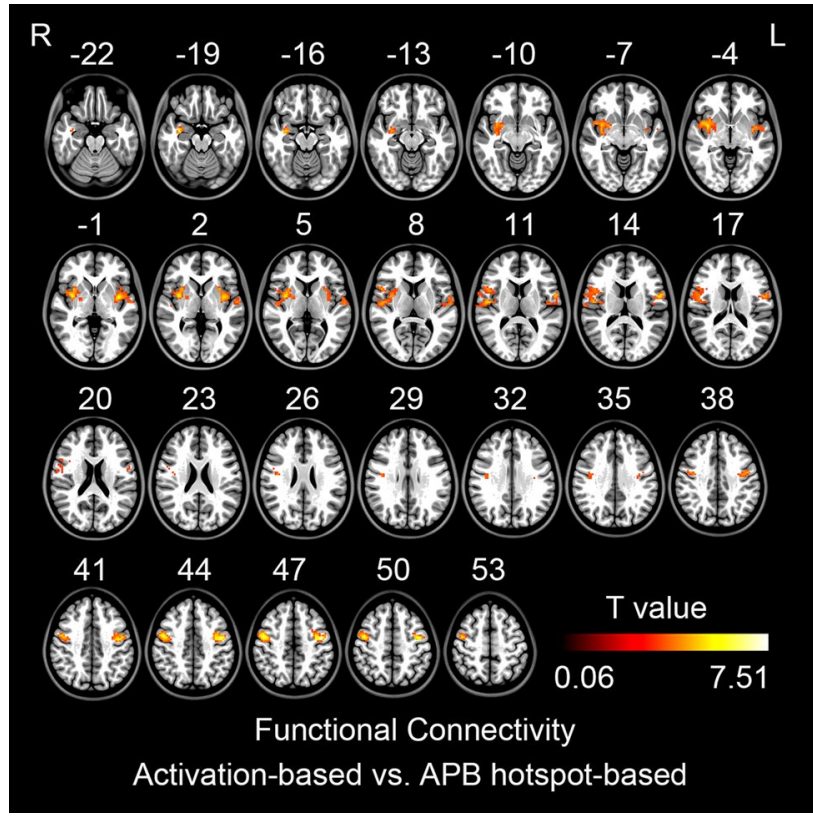

Fig. 7 The differences between activation-based and APB hotspot-based functional connectivity (GRF correction, single voxel $\mathrm{p}<0.001$, cluster level $\mathrm{p}<0.05$ ). The warm color indicates more intensive activation-based functional connectivity than APB hotspotbased functional connectivity. L, left hemisphere; R, right hemisphere with the previous studies in which the authors also found the location of fMRI activation is lateral to the TMS hotspot (Diekhoff et al. 2011; Sparing et al. 2008). Moreover, some other studies found that the TMS hotspot location was more anterior to the fMRI activation (Bastings et al. 1998; Herwig et al. 2002; Lotze et al. 2003b). Many factors may have contributed such mismatch. The exact fMRI tasks varied across studies, such as the mode of movement (visual guided or self-paced), number of fingers (thumb movement solely or thumb movement against to each of the four other fingers, or "hand clenching") (Bastings et al. 1998; Boroojerdi et al. 1999; Diekhoff et al. 2011; Herwig et al. 2002; Sparing et al. 2008). The parameters of TMS hotspot may vary across studies, e.g., the coil orientation. Although all these TMS studies placed the coil over the motor area with $45^{\circ}$ against the middle line of brain with the handle backwards, the actual current orientation to the neurons highly depends on individual neuroanatomy and tissue anisotropy (Rossini et al. 2015). Such mismatch may also be due to a fact that the fMRI motor tasks are much more complicated than TMSinduced finger movement. Compared with TMS-induced finger movement, the finger tapping fMRI task involves more voluntary movement. A previous fMRI study compared voluntary movement and non-voluntary movement and found that the voluntary movement activation peak voxel sat more anteriorly and laterally in the contralateral primary motor area (Lotze et al. 2003a), being consistent with the present study (TMS-induced finger movement can be considered as 
Table 5 The differences between activation-based and APB hotspot-based functional connectivity

\begin{tabular}{|c|c|c|c|c|c|c|c|}
\hline \multirow{2}{*}{$\begin{array}{l}\text { Brain region } \\
\text { Right Hippocampus (extend to right puta- } \\
\text { men, right globus pallidus and right } \\
\text { insula) }\end{array}$} & \multirow{2}{*}{$\begin{array}{l}\text { Brodmann area } \\
20,48\end{array}$} & \multicolumn{3}{|c|}{$\begin{array}{l}\text { Montreal Neurologi- } \\
\text { cal Institute (X Y Z) }\end{array}$} & \multirow{2}{*}{$\begin{array}{l}\text { Cluster size }\left(\mathrm{mm}^{3}\right) \\
12,555\end{array}$} & \multirow{2}{*}{$\begin{array}{l}\text { T value } \\
6.19\end{array}$} & \multirow{2}{*}{$\begin{array}{l}\text { Peak voxel p value } \\
<0.001\end{array}$} \\
\hline & & 36 & -6 & 18 & & & \\
\hline Left Insula (extend to left putamen) & 48 & -39 & 0 & 0 & 5670 & 5.63 & $<0.001$ \\
\hline Right Precentral Gyrus & 6 & 45 & -6 & 51 & 3969 & 6.33 & $<0.001$ \\
\hline Left Precentral Gyrus & 6 & -45 & -6 & 48 & 3375 & 7.54 & $<0.001$ \\
\hline
\end{tabular}

$A P B$ abductor pollicis brevis

non-voluntary movement). Therefore, the mismatch locations between finger tapping fMRI activation and TMS hotspot might be partly due to voluntary vs. non-voluntary movements. There might be also other components contributed to the location differences derived from visual cue that informed the participant to move. Non-human primate study found that a category of neurons responds to an object during observation without any movement, called visuomotor neurons (Raos et al. 2004). However, fMRI could not discern whether the activation of finger tapping task with a visual cue is contributed by this category of neurons. Even so, fMRI studies reported the premotor area responds to visual input, which codes information during the movement preparation (Gallivan et al. 2013). The premotor area involves in decoding of familiar objects and planning a motor action (Smith and Goodale 2015). fMRI activation location relates to more cognitive and voluntary elements of movement than TMS hotspot location.

\section{Different Functional Connectivity for Finger Tapping fMRI Activation and TMS Hotspot}

We found that the finger tapping fMRI activation sat more laterally than TMS hotspot (Tables 1, 2 and 3). We were then interested in their difference of functional connectivity. Paired t-tests showed that the finger tapping activation seed had significantly more intensive functional connectivity than TMS hotspot seed. These brain areas included the bilateral putamen, right globus pallidus, bilateral insula, and bilateral premotor area (Fig. 7, Table 5). No any significant more intensive functional connectivity was found for the TMS hotspot seed than the finger tapping activation seed.

Premotor area has been widely reported to be involved in cognitive processing for movement. Motor preparation and execution are two stages of voluntary movements, involving $\mathrm{M} 1$, dorsal premotor cortex (PMd), ventral premotor (PMv) and supplementary motor area (Chouinard and Paus 2006; Gallivan et al. 2013, 2011a, b; Hirose et al. 2018; Nambu et al. 2015). A previous TMS study showed that, after lowfrequency repetitive TMS applied over PMd, subjects could not select appropriate weight from two different weights to lift based on the visual cues (Chouinard et al. 2005). The current findings of more intensive functional connectivity of bilateral premotor areas with the finger tapping task activation than with TMS hotspot were in line with the comprehensive function of premotor area mentioned above.

Basal ganglia are key node in the motor circuits. The posterior (sensorimotor) putamen had been observed in finger movement task (Lehericy et al. 2005). And putamen was involved in the motor loop circuit (Middleton and Strick 2000). In our previous meta-analysis study, decreased amplitude low frequency fluctuations (ALFF) were observed in bilateral putamen in Parkinson's disease patients (Wang et al. 2018). In the present study, the intensive functional connectivity was revealed at left posterior putamen, right posterior and middle putamen-globus pallidus with the activation seed (Fig. 6, Table 5). The current results suggest that the finger tapping activation area is more related to motor circuits than the TMS hotspot.

\section{Potential Applications of fMRI-Guided TMS Treatment}

The present study found that the finger tapping activation and TMS hotspot located differently in the motor cortex. Moreover, the finger tapping activation peak had more intensive functional connectivity with bilateral premotor area, bilateral insula, bilateral putamen and right globus pallidus. RS-fMRI functional connectivity studies have helped understand the complex mechanism of rTMS modulation effect on the brain activity. Some studies reported that the rTMS could modulate the brain network or functional connectivity (Andoh et al. 2015; Chen et al. 2013; Cocchi et al. 2016; Cocchi et al. 2015; Eldaief et al. 2011; Halko et al. 2014; Ji et al. 2017; Nettekoven et al. 2014, 2015; Wang et al. 2014; Watanabe et al. 2014). The current results of more intensive functional connectivity of finger tapping activation area than the TMS hotspot may suggest that TMS treatment targeting on the fMRI activation area might result in more remote effects than targeting on the hotspot. Therefore, the fMRI 
activation might be a more ideal target for improvement of motor functions or treatment of movement disorders. To our knowledge, we conducted the first study to demonstrate the different brain networks of motor task fMRI activation vs. TMS hotspot area. One clinical importance of our findings is that rTMS treatment on movement disorders may take the motor task fMRI activation area, but not the TMS hotspot, as individual and precise stimulation target. Although the TMS hotspot is also an individualized and precise stimulation target, this target may have less widespread effect on remote brain regions, because TMS hotspot region has much less intensive brain network than motor fMRI task activation area.

\section{Conclusion}

Finger tapping fMRI activation location differs from the TMS hotspot and has more intensive functional connectivity with motor-related brain regions, e.g., premotor area and basal ganglia. The current results might be of clinical importance for rTMS treatment target selection. Future rTMS studies could take the motor task fMRI activation area as individual and precise stimulation target for treatment on movement disorders.

\section{Limitations}

A few limitations should be addressed. (1) Repetitive TMS was not performed, therefore, it is not known whether rTMS could differently modulate the hotspot network and motor task activation network; (2) We speculated that the hotspot may be more related to passive movement, however, due to the limitations of the experimental equipment, the present study did not measure the passive movement in fMRI. (3) It has been reported that the fMRI activation peak could be different if different fMRI imaging sequences were used (e.g., spin-echo vs. gradient-echo) (Diekhoff et al. 2011), future studies should take this issue into account.

Acknowledgements This work was supported by the National Natural Science Foundation of China (Nos. 81701776, 31471084 and 81520108016).

Authors Contributions WJ searched literature, performed the data analysis and generated the images, and wrote the manuscript. HM, YJ, HW, XD, ZF and NZ recruited the subjects, collected the data. GJ assisted with the data analysis.
$\mathrm{YZ}$ and $\mathrm{JZ}$ contributed to the experimental design, revised the article, and developed the research concept.

\section{Compliance with Ethical Standards}

Conflict of interests The authors declare that they have no conflicts of interest.

Ethical Approval All procedures performed in studies involving human participants were in accordance with the ethical standards of the institutional and national research committee (the Ethics Committee of the Center for Cognition and Brain Disorders (CCBD) at Hangzhou Normal University (HZNU), 20170405) and with the 1964 Helsinki declaration and its later amendments or comparable ethical standards.

Open Access This article is distributed under the terms of the Creative Commons Attribution 4.0 International License (http://creativeco mmons.org/licenses/by/4.0/), which permits unrestricted use, distribution, and reproduction in any medium, provided you give appropriate credit to the original author(s) and the source, provide a link to the Creative Commons license, and indicate if changes were made.

\section{References}

Ahdab R, Ayache SS, Brugieres P, Farhat WH, Lefaucheur JP (2016) The hand motor hotspot is not always located in the hand knob: a neuronavigated transcranial magnetic stimulation study. Brain Topogr 29:590-597. https://doi.org/10.1007/s10548-016-0486-2

Andoh J, Matsushita R, Zatorre RJ (2015) Asymmetric interhemispheric transfer in the auditory network: evidence from TMS, resting-state fMRI, and diffusion imaging. J Neurosci 35:1460214611. https://doi.org/10.1523/JNEUROSCI.2333-15.2015

Bastings EP et al (1998) Co-registration of cortical magnetic stimulation and functional magnetic resonance imaging. NeuroReport 9:1941-1946. https://doi.org/10.1097/00001756-19980 6220-00005

Benninger DH et al (2009) Safety study of $50 \mathrm{~Hz}$ repetitive transcranial magnetic stimulation in patients with Parkinson's disease. Clin Neurophysiol 120:809-815. https://doi.org/10.1016/j.clinp h.2009.01.012

Benninger DH et al (2011) Intermittent theta-burst transcranial magnetic stimulation for treatment of Parkinson disease. Neurology 76:601-609. https://doi.org/10.1212/WNL.0b013e31820ce6bb

Boroojerdi B, Foltys H, Krings T, Spetzger U, Thron A, Topper R (1999) Localization of the motor hand area using transcranial magnetic stimulation and functional magnetic resonance. Clin Neurophysiol 110:699-704

Chen AC et al (2013) Causal interactions between fronto-parietal central executive and default-mode networks in humans. Proc Natl Acad Sci USA 110:19944-19949. https://doi.org/10.1073/ pnas. 1311772110

Chouinard PA, Paus T (2006) The primary motor and premotor areas of the human cerebral cortex. Neuroscientist 12:143-152. https:// doi.org/10.1177/1073858405284255

Chouinard PA, Leonard G, Paus T (2005) Role of the primary motor and dorsal premotor cortices in the anticipation of forces during object lifting. J Neurosci 25:2277-2284. https://doi.org/10.1523/ JNEUROSCI.4649-04.2005 
Cocchi L, Sale MV, Lord A, Zalesky A, Breakspear M, Mattingley JB (2015) Dissociable effects of local inhibitory and excitatory thetaburst stimulation on large-scale brain dynamics. J Neurophysiol 113:3375-3385. https://doi.org/10.1152/jn.00850.2014

Cocchi L et al (2016) A hierarchy of timescales explains distinct effects of local inhibition of primary visual cortex and frontal eye fields. eLife. https://doi.org/10.7554/elife. 15252

Cole DM, Smith SM, Beckmann CF (2010) Advances and pitfalls in the analysis and interpretation of resting-state FMRI data. Front Syst Neurosci 4:8. https://doi.org/10.3389/fnsys.2010.00008

Diekhoff S, Uludag K, Sparing R, Tittgemeyer M, Cavusoglu M, von Cramon DY, Grefkes C (2011) Functional localization in the human brain: gradient-Echo, Spin-Echo, and arterial spin-labeling fMRI compared with neuronavigated TMS. Hum Brain Mapp 32:341-357. https://doi.org/10.1002/hbm.21024

Diekhoff-Krebs S, Pool EM, Sarfeld AS, Rehme AK, Eickhoff SB, Fink GR, Grefkes C (2017) Interindividual differences in motor network connectivity and behavioral response to iTBS in stroke patients. NeuroImage Clin 15:559-571. https://doi. org/10.1016/j.nicl.2017.06.006

Eldaief MC, Halko MA, Buckner RL, Pascual-Leone A (2011) Transcranial magnetic stimulation modulates the brain's intrinsic activity in a frequency-dependent manner. Proc Natl Acad Sci USA 108:21229-21234. https://doi.org/10.1073/pnas.11131 03109

Fox MD, Snyder AZ, Vincent JL, Corbetta M, Van Essen DC, Raichle ME (2005) The human brain is intrinsically organized into dynamic, anticorrelated functional networks. Proc Natl Acad Sci USA 102:9673-9678. https://doi.org/10.1073/pnas.0504136102

Gallivan JP, McLean DA, Smith FW, Culham JC (2011a) Decoding effector-dependent and effector-independent movement intentions from human parieto-frontal brain activity. J Neurosci 31:1714917168. https://doi.org/10.1523/JNEUROSCI.1058-11.2011

Gallivan JP, McLean DA, Valyear KF, Pettypiece CE, Culham JC (2011b) Decoding action intentions from preparatory brain activity in human parieto-frontal networks. J Neurosci 31:9599-9610. https://doi.org/10.1523/JNEUROSCI.0080-11.2011

Gallivan JP, McLean DA, Flanagan JR, Culham JC (2013) Where one hand meets the other: limb-specific and action-dependent movement plans decoded from preparatory signals in single human frontoparietal brain areas. J Neurosci 33:1991-2008. https://doi. org/10.1523/JNEUROSCI.0541-12.2013

Geyer S et al (1996) Two different areas within the primary motor cortex of man. Nature 382:805-807. https://doi.org/10.1038/38280 $5 \mathrm{a} 0$

Halko MA, Farzan F, Eldaief MC, Schmahmann JD, Pascual-Leone A (2014) Intermittent theta-burst stimulation of the lateral cerebellum increases functional connectivity of the default network. J Neurosci 34:12049-12056. https://doi.org/10.1523/JNEUR OSCI.1776-14.2014

Hampson M, Peterson BS, Skudlarski P, Gatenby JC, Gore JC (2002) Detection of functional connectivity using temporal correlations in MR images. Hum Brain Mapp 15:247-262

Hanlon CA, DeVries W, Dowdle LT, West JA, Siekman B, Li X, George MS (2015) A comprehensive study of sensorimotor cortex excitability in chronic cocaine users: integrating TMS and functional MRI data. Drug Alcohol Depend 157:28-35. https:// doi.org/10.1016/j.drugalcdep.2015.07.1196

Havrankova P et al (2010) Repetitive TMS of the somatosensory cortex improves writer's cramp and enhances cortical activity. Neuroendocrinol Lett 31:73-86

Herwig U, Kolbel K, Wunderlich AP, Thielscher A, von Tiesenhausen C, Spitzer M, Schonfeldt-Lecuona C (2002) Spatial congruence of neuronavigated transcranial magnetic stimulation and functional neuroimaging. Clin Neurophysiol 113:462-468
Hirose S, Nambu I, Naito E (2018) Cortical activation associated with motor preparation can be used to predict the freely chosen effector of an upcoming movement and reflects response time: an fMRI decoding study. NeuroImage 183:584-596. https://doi. org/10.1016/j.neuroimage.2018.08.060

Hulst HE et al (2017) rTMS affects working memory performance, brain activation and functional connectivity in patients with multiple sclerosis. J Neurol, Neurosurg Psychiatry 88:386-394. https ://doi.org/10.1136/jnnp-2016-314224

Ji GJ, Yu F, Liao W, Wang K (2017) Dynamic aftereffects in supplementary motor network following inhibitory transcranial magnetic stimulation protocols. NeuroImage 149:285-294. https://doi. org/10.1016/j.neuroimage.2017.01.035

Kallioniemi E, Julkunen P (2016) Alternative stimulation intensities for mapping cortical motor area with navigated TMS. Brain Topogr 29:395-404. https://doi.org/10.1007/s10548-016-0470-x

Khedr EM, Farweez HM, Islam H (2003) Therapeutic effect of repetitive transcranial magnetic stimulation on motor function in Parkinson's disease patients. Eur J Neurol 10:567-572

Khedr EM, Rothwell JC, Shawky OA, Ahmed MA, Hamdy A (2006) Effect of daily repetitive transcranial magnetic stimulation on motor performance in Parkinson's disease. Mov Disord 21:2201-2205

Lefaucheur JP (2019) Transcranial magnetic stimulation. Handb Clin Neurol 160:559-580. https://doi.org/10.1016/B978-0-444-64032 $-1.00037-0$

Lehericy S, Benali H, Van de Moortele PF, Pelegrini-Issac M, Waechter T, Ugurbil K, Doyon J (2005) Distinct basal ganglia territories are engaged in early and advanced motor sequence learning. Proc Natl Acad Sci USA 102:12566-12571. https://doi.org/10.1073/ pnas.0502762102

Lotze M, Braun C, Birbaumer N, Anders S, Cohen LG (2003a) Motor learning elicited by voluntary drive. Brain 126:866-872

Lotze M, Kaethner RJ, Erb M, Cohen LG, Grodd W, Topka H (2003b) Comparison of representational maps using functional magnetic resonance imaging and transcranial magnetic stimulation. Clin Neurophysiol 114:306-312

Marsili L et al (2017) BDNF and LTP-/LTD-like plasticity of the primary motor cortex in Gilles de la Tourette syndrome. Exp Brain Res 235:841-850. https://doi.org/10.1007/s00221-016-4847-6

Middleton FA, Strick PL (2000) Basal ganglia and cerebellar loops: motor and cognitive circuits Brain research. Brain Res Rev $31: 236-250$

Nambu I, Hagura N, Hirose S, Wada Y, Kawato M, Naito E (2015) Decoding sequential finger movements from preparatory activity in higher-order motor regions: a functional magnetic resonance imaging multi-voxel pattern analysis. Eur J Neurosci 42:28512859. https://doi.org/10.1111/ejn.13063

Nettekoven C et al (2014) Dose-dependent effects of theta burst rTMS on cortical excitability and resting-state connectivity of the human motor system. J Neurosci 34:6849-6859. https://doi.org/10.1523/ JNEUROSCI.4993-13.2014

Nettekoven $C$ et al (2015) Inter-individual variability in cortical excitability and motor network connectivity following multiple blocks of rTMS. NeuroImage 118:209-218. https://doi.org/10.1016/j. neuroimage.2015.06.004

Pitkanen M, Kallioniemi E, Julkunen P (2015) Extent and location of the excitatory and inhibitory cortical hand representation maps: a navigated transcranial magnetic stimulation study. Brain Topogr 28:657-665. https://doi.org/10.1007/s10548-015-0442-6

Puce A et al (1995) Functional magnetic resonance imaging of sensory and motor cortex: comparison with electrophysiological localization. J Neurosurg 83:262-270. https://doi.org/10.3171/ jns.1995.83.2.0262 
Raos V, Umilta MA, Gallese V, Fogassi L (2004) Functional properties of grasping-related neurons in the dorsal premotor area F2 of the macaque monkey. J Neurophysiol 92:1990-2002. https://doi. org/10.1152/jn.00154.2004

Rossini PM et al (2015) Non-invasive electrical and magnetic stimulation of the brain, spinal cord, roots and peripheral nerves: basic principles and procedures for routine clinical and research application. An updated report from an I.F.C.N. Comm Clin Neurophysiol 126:1071-1107. https://doi.org/10.1016/j.clinph.2015.02.001

Siebner HR, Rossmeier C, Mentschel C, Peinemann A, Conrad B (2000) Short-term motor improvement after sub-threshold 5-Hz repetitive transcranial magnetic stimulation of the primary motor hand area in Parkinson's disease. J Neurol Sci 178:91-94

Smith FW, Goodale MA (2015) Decoding visual object categories in early somatosensory cortex. Cereb Cortex 25:1020-1031. https:// doi.org/10.1093/cercor/bht292

Sparing R, Buelte D, Meister IG, Paus T, Fink GR (2008) Transcranial magnetic stimulation and the challenge of coil placement: a comparison of conventional and stereotaxic neuronavigational strategies. Hum Brain Mapp 29:82-96. https://doi.org/10.1002/ hbm. 20360

Stinear CM, Barber PA, Coxon JP, Verryt TS, Acharya PP, Byblow WD (2009) Repetitive stimulation of premotor cortex affects primary motor cortex excitability and movement preparation. Brain Stimul 2:152-162. https://doi.org/10.1016/j.brs.2009.01.001

Van Dijk KR, Hedden T, Venkataraman A, Evans KC, Lazar SW, Buckner RL (2010) Intrinsic functional connectivity as a tool for human connectomics: theory, properties, and optimization. J Neurophysiol 103:297-321. https://doi.org/10.1152/jn.00783.2009

Vitikainen AM, Salli E, Lioumis P, Makela JP, Metsahonkala L (2013) Applicability of nTMS in locating the motor cortical representation areas in patients with epilepsy. Acta Neurochir 155:507-518. https://doi.org/10.1007/s00701-012-1609-5
Wagle Shukla A, Shuster JJ, Chung JW, Vaillancourt DE, Patten C, Ostrem J, Okun MS (2016) Repetitive transcranial magnetic stimulation (rTMS) therapy in Parkinson disease: a meta-analysis. PM \& R. 8:356-366. https://doi.org/10.1016/j.pmrj.2015.08.009

Wang JX et al (2014) Targeted enhancement of cortical-hippocampal brain networks and associative memory. Science 345:1054-1057. https://doi.org/10.1126/science. 1252900

Wang J, Zhang JR, Zang YF, Wu T (2018) Consistent decreased activity in the putamen in Parkinson's disease: a meta-analysis and an independent validation of resting-state fMRI. GigaScience. https ://doi.org/10.1093/gigascience/giy071

Watanabe $\mathrm{T}$ et al (2014) Bidirectional effects on interhemispheric resting-state functional connectivity induced by excitatory and inhibitory repetitive transcranial magnetic stimulation. Hum Brain Mapp 35:1896-1905. https://doi.org/10.1002/hbm.22300

Yan CG et al (2013) A comprehensive assessment of regional variation in the impact of head micromovements on functional connectomics. NeuroImage 76:183-201. https://doi.org/10.1016/j. neuroimage.2013.03.004

Yan CG, Wang XD, Zuo XN, Zang YF (2016) DPABI: data processing $\&$ analysis for (resting-state) brain imaging. Neuroinformatics 14:339-351. https://doi.org/10.1007/s12021-016-9299-4

Yousry TA, Schmid UD, Alkadhi H, Schmidt D, Peraud A, Buettner A, Winkler P (1997) Localization of the motor hand area to a knob on the precentral gyrus. A new landmark. Brain 120(Pt 1):141-157

Zhang D et al (2009) Preoperative sensorimotor mapping in brain tumor patients using spontaneous fluctuations in neuronal activity imaged with functional magnetic resonance imaging: initial experience. Neurosurgery 65:226-236. https://doi.org/10.1227/01. NEU.0000350868.95634.CA

Publisher's Note Springer Nature remains neutral with regard to jurisdictional claims in published maps and institutional affiliations. 